\title{
Convergence of the solution of an impulsive differential equation with piecewise constant argument
}

\author{
Huseyin Bereketoglu and Gizem S. Oztepe
}




\title{
CONVERGENCE OF THE SOLUTION OF AN IMPULSIVE DIFFERENTIAL EQUATION WITH PIECEWISE CONSTANT ARGUMENTS
}

\author{
HUSEYIN BEREKETOGLU AND GIZEM S. OZTEPE
}

Received 18 September, 2012

Abstract. We show the existence of the unique solution of impulsive differential equation

$$
\left\{\begin{array}{c}
x^{\prime}(t)=a(t)(x(t)-x(\lfloor t-1\rfloor))+f(t), t \neq n \in \mathbb{Z}^{+}=\{1,2, \ldots\}, t \geq 0, \\
\Delta x(t)=c_{t} x(t)+d_{t}, t=n \in \mathbb{Z}^{+},
\end{array}\right.
$$

with the initial conditions

$$
x(-1)=x_{-1}, x(0)=x_{0},
$$

where $\lfloor$.$\rfloor denotes the floor integer function. Moreover, we obtain sufficient conditions for the$ asymptotic constancy of this equation and we compute, as $t \rightarrow \infty$, the limits of the solutions of the impulsive equation with $c_{n}=0$ in terms of the initial conditions, a special solution of the corresponding adjoint equation and a solution of the corresponding difference equation.

2010 Mathematics Subject Classification: 34K06; 34K45

Keywords: asymptotic constancy, differential equation with piecewise constant argument, impulsive differential equation

\section{INTRODUCTION}

The theory of differential equations with piecewise constant arguments (DEP-CA) of the type

$$
x^{\prime}(t)=f(t, x(t), x(h(t)))
$$

was initiated in $[14,40]$ where $h(t)=\lfloor t\rfloor,\lfloor t-n\rfloor,\lfloor t+n\rfloor$, etc. and $\lfloor$.$\rfloor denotes the$ floor integer function. These types of equations have been intensively investigated for twenty five years. Systems described by DEPCA exist in a large area such as biomedicine, chemistry, physics and mechanical engineering. Busenberg and Cooke [13] first established a mathematical model with a piecewise constant argument for analyzing vertically transmitted diseases. Examples in practice include machinery driven by servo units, charged particles moving in a piecewise constantly varying electric field and elastic systems impelled by a Geneva wheel.

$D E P C A$ are also closely related to difference and differential equations. So, they describe hybrid dynamical systems and combine the properties of both differential 
and difference equations. The oscillation, periodicity and some asymptotic properties of various differential equations with piecewise constant arguments were methodically demonstrated in [1-5, 26-28, 35, 36, 38, 42]. Also, Wiener's book [43] is a distinguished source in this area.

Impulsive differential equations are a basic tool to study the dynamics of processes that are subjected to abrupt changes in their states. Theory of impulsive differential equations has been motivated by a number of applied problems such as control theory [24,25], population dynamics [37], chemotherapeutic treatment in medicine [31] and some physics problems [32]. A significant development has been made in the mathematical theory of impulsive differential equations in the last two decades; see the monographs [7,39].

But, there are only a few papers on impulsive differential equations with piecewise constant arguments (IDEPCA) [9, 29,33,44]. In [33], Li and Shen considered the problem

$$
\begin{aligned}
y^{\prime}(t) & =f(t, y\lfloor t-k\rfloor), t \neq n, t \in J, \\
\Delta y\left(n^{+}\right) & =I_{n}(y(n)), n=1,2, \ldots, p, y(0)=y(T) .
\end{aligned}
$$

Using the method of upper and lower solutions, they proved that it has at least one solution. In [44], Wiener and Lakshmikantham established the existence and uniqueness of solutions of the initial value problem

$$
x^{\prime}(t)=f(x(t), x(g(t))), x(0)=x_{0},
$$

and they also studied the cases of oscillation and stability, where $f$ is a continuous function and $g:[0, \infty) \rightarrow[0, \infty), g(t) \leq t$, is a step function. In [9] and [29], some qualitative aspects of advanced and delay IDEPCA are investigated. In [34], the authors investigated the impulsive stabilization of certain delay differential equations with piecewise constant argument by using Lyapunov function and analysis methods. They showed that some nonimpulsive systems can be stabilized by imposition of impulsive controls.

Lately, the problem of the asymptotic constancy of solutions was studied for some functional differential equations $[6,8,11,12,15-23,41]$ and as well the same problem has been considered for some impulsive delay differential equations [10,30]. So, due to the practical reasons and the papers mentioned above one can be motivated to deal with the problem of asymptotic constancy of solutions of an impulsive differential equation with piecewise constant arguments.

In this paper, we consider the first order nonhomogeneous linear impulsive differential equation with piecewise constant argument

$$
\begin{gathered}
x^{\prime}(t)=a(t)(x(t)-x(\lfloor t-1\rfloor))+f(t), t \neq n \in \mathbb{Z}^{+}, t \geq 0, \\
\Delta x(t)=c_{t} x(t)+d_{t}, t=n \in \mathbb{Z}^{+},
\end{gathered}
$$


with the initial conditions

$$
x(-1)=x_{-1}, x(0)=x_{0},
$$

where $a(t)$ and $f(t)$ are continuous real valued functions on $[0, \infty), c_{n} \in \mathbb{R} \backslash\{1\}$, $d_{n} \in \mathbb{R}, n \in \mathbb{Z}^{+}, \mathbb{Z}^{+}=\{1,2, \ldots\}, x_{0}, x_{-1} \in \mathbb{R}, \Delta x(n)=x\left(n^{+}\right)-x\left(n^{-}\right)$, $x\left(n^{+}\right)=\lim _{t \rightarrow n^{+}} x(t), x\left(n^{-}\right)=\lim _{t \rightarrow n^{-}} x(t)$ and $\lfloor$.$\rfloor denotes the floor integer function.$

The main purpose of this work is to obtain sufficient conditions for asymptotic constancy of the solution $x(t)$ of (1.1) - (1.3) and also, as $t \rightarrow \infty$, to compute the limit of the solution of the impulsive differential equation with piecewise constant argument

$$
\begin{gathered}
x^{\prime}(t)=a(t)(x(t)-x(\lfloor t-1\rfloor))+f(t), t \neq n \in \mathbb{Z}^{+}, t \geq 0, \\
\Delta x(t)=d_{t}, t=n \in \mathbb{Z}^{+},
\end{gathered}
$$

in terms of initial conditions, the solution of an integral equation and the solution of a corresponding difference equation. To the best of author's knowledge, this problem has not been studied yet.

\section{EXISTENCE OF SOLUTIONS}

Definition 1. A function $x(t)$ defined on $[0, \infty)$ is said to be a solution of $(1.1)-$ (1.3) if it satisfies the following conditions:

$\left(d_{1}\right) x:[0, \infty) \rightarrow \mathbb{R}$ is continuous with the possible exception of the points $t \in \mathbb{Z}^{+}$, $\left(d_{2}\right) x(t)$ is right continuous and has left-hand limits at the points $t \in \mathbb{Z}^{+}$,

$\left(d_{3}\right) x^{\prime}(t)$ exists for every $t \in[0, \infty)$ with the possible exception of the points $t \in \mathbb{Z}^{+}$where one-sided derivatives exist,

$\left(d_{4}\right) x(t)$ satisfies $(1.1)$ for any $t \in(0, \infty)$ with the possible exception of the points $t \in \mathbb{Z}^{+}$,

$\left(d_{5}\right) x(t)$ satisfies (1.2) for every $t=n \in \mathbb{Z}^{+}$,

$\left(d_{6}\right) x(-1)=x_{-1}, x(0)=x_{0}$.

Theorem 1. The initial value problem (1.1) - (1.3) has a unique solution $x(t)$ on $[0, \infty)$

$$
\begin{aligned}
x(t) & =\exp \left(\int_{\lfloor t\rfloor}^{t} a(u) d u\right) z_{\lfloor t\rfloor}+\left(1-\exp \left(\int_{\lfloor t\rfloor}^{t} a(u) d u\right)\right) z_{\lfloor t\rfloor-1} \\
& +\int_{\lfloor t\rfloor}^{t} \exp \left(\int_{s}^{t} a(u) d u\right) f(s) d s
\end{aligned}
$$


where $\lfloor$.$\rfloor denotes the floor integer function, z_{\lfloor t\rfloor}=x(\lfloor t\rfloor)$ for $t \in[0, \infty)$ and it is a solution of the corresponding difference equation

$$
\begin{aligned}
z_{\lfloor t\rfloor+1} & =\left(\left(1-c_{\lfloor t\rfloor+1}\right)^{-1} \exp \left(\int_{\lfloor t\rfloor}^{\lfloor t\rfloor+1} a(u) d u\right)\right) z_{\lfloor t\rfloor} \\
& +\left(1-c_{\lfloor t\rfloor+1}\right)^{-1}\left(1-\exp \left(\int_{\lfloor t\rfloor}^{\lfloor t\rfloor+1} a(u) d u\right)\right) z_{\lfloor t\rfloor-1} \\
& +\left(1-c_{\lfloor t\rfloor+1}\right)^{-1}\left(\int_{\lfloor t\rfloor}^{\lfloor t\rfloor+1} \exp \left(\int_{s}^{\lfloor t\rfloor+1} a(u) d u\right) f(s) d s+d_{\lfloor t\rfloor+1}\right) .
\end{aligned}
$$

Proof. Let $x(t)$ be a solution of (1.1) on $n \leq t<n+1$. Then, Eq.(1.1) reduces to linear ordinary differential equation

$$
x^{\prime}(t)=a(t)(x(t)-x(n-1))+f(t) .
$$

When we solve this equation, we have

$$
\begin{aligned}
x(t) & =\exp \left(\int_{n}^{t} a(u) d u\right) x(n)+\left(1-\exp \left(\int_{n}^{t} a(u) d u\right)\right) x(n-1) \\
& +\int_{n}^{t} \exp \left(\int_{s}^{t} a(u) d u\right) f(s) d s, n \leq t<n+1 .
\end{aligned}
$$

If we denote the solution $x(t)$ defined by (2.3) as $x_{n}(t)$, then $x_{n+1}(t)$ implies the solution of Eq.(1.1) on the interval $n+1 \leq t<n+2$ :

$$
\begin{aligned}
x_{n+1}(t) & =\exp \left(\int_{n+1}^{t} a(u) d u\right) x(n+1)+\left(1-\exp \left(\int_{n+1}^{t} a(u) d u\right)\right) x(n) \\
& +\int_{n+1}^{t} \exp \left(\int_{s}^{t} a(u) d u\right) f(s) d s, n+1 \leq t<n+2
\end{aligned}
$$

Using impulse conditions (1.2) for $t=n+1$, we have

$$
\Delta x(n+1)=c_{n+1} x(n+1)+d_{n+1},
$$

that is,

$$
x(n+1)^{+}-x(n+1)^{-}=c_{n+1} x(n+1)+d_{n+1} .
$$


Since $x(t)$ is right continuous, the previous equality reduces to

$$
x_{n}\left((n+1)^{-}\right)=\left(1-c_{n+1}\right) x_{n+1}(n+1)-d_{n+1} .
$$

Considering this equality together with (2.3) and (2.4), we obtain the second order nonhomogeneous difference equation

$$
\begin{aligned}
x(n+1) & =\left(1-c_{n+1}\right)^{-1} \exp \left(\int_{n}^{n+1} a(u) d u\right) x(n) \\
& +\left(1-c_{n+1}\right)^{-1}\left(1-\exp \left(\int_{n}^{n+1} a(u) d u\right)\right) x(n-1) \\
& +\left(1-c_{n+1}\right)^{-1}\left(\int_{n}^{n+1} \exp \left(\int_{s}^{n+1} a(u) d u\right) f(s) d s+d_{n+1}\right) .
\end{aligned}
$$

Let us define $x(\lfloor t\rfloor)=z_{\lfloor t\rfloor}$ for $t \in[0, \infty)$. So, $x(n)=z_{n}$ for $n=0,1,2, \ldots$, and Eq.(2.5) can be rewritten as

$$
\begin{aligned}
z_{n+1} & =\left(1-c_{n+1}\right)^{-1} \exp \left(\int_{n}^{n+1} a(u) d u\right) z_{n} \\
& +\left(1-c_{n+1}\right)^{-1}\left(1-\exp \left(\int_{n}^{n+1} a(u) d u\right)\right) z_{n-1} \\
& +\left(1-c_{n+1}\right)^{-1}\left(\int_{n}^{n+1} \exp \left(\int_{s}^{n+1} a(u) d u\right) f(s) d s+d_{n+1}\right)
\end{aligned}
$$

which implies Eq.(2.2) for $t \in[0, \infty)$.

Taking into account the initial conditions $x(-1)=x_{-1}=z_{-1}$ and $x(0)=x_{0}=$ $z_{0}$, the solution of difference equation (2.6) is obtained uniquely. So, the unique solution of $(1.1)-(1.3)$ can be written as $(2.1)$ on $[0, \infty)$.

In this while, we note the following statements:

a) Along this paper we will assume that all solutions of the difference equation (2.6) are bounded, that is, for any solution $z_{n}$ of (2.6) there is a real positive constant $L$ such that

$$
\left|z_{n}\right| \leq L, n \in \mathbb{Z}^{+}
$$


b) A straightforward verification shows that the solution of the initial value problem (1.1) - (1.3) satisfies the following integral equation

$x(t)=\left\{\begin{array}{c}x_{-1}, t=-1, \\ x_{0}+\int_{0}^{t} a(s) x(s) d s-\int_{0}^{t} a(s) x(\lfloor s-1\rfloor) d s+\int_{0}^{t} f(s) d s+\sum_{i=1}^{\lfloor t\rfloor} c_{i} z_{i}+\sum_{i=1}^{\lfloor t\rfloor} d_{i}, \\ t \geq 0,\end{array}\right.$

where $z_{i}=x(i), i=1,2, \ldots,\lfloor t\rfloor$.

\section{MAIN RESUltS}

This section contains the statements of our main results.

Theorem 2. Let $a(t)$ and $f(t)$ be continuous functions on the interval $[0, \infty)$, $c: \mathbb{Z}^{+} \longrightarrow \backslash\{1\}, d: \mathbb{Z}^{+} \longrightarrow$ and $n \in \mathbb{Z}^{+}$.

If

(i) $\int_{0}^{\infty}|a(s)| d s \leq K_{1}<\infty$,

(ii) $\int_{0}^{\infty}|f(s)| d s \leq K_{2}<\infty$,

(ii i ) $\prod_{i=1}^{\infty}\left(1+\left|c_{i}\right|\right) \leq L_{1}<\infty$

(iv) $\sum_{i=1}^{\infty}\left|d_{i}\right| \leq L_{2}<\infty$,

then the solution $x(t)$ of $(1.1)-(1.3)$ tends to a constant as $t \rightarrow \infty$, where $K_{j}, L_{j}$, $j=1,2$, are real positive constants.

Theorem 3. Suppose that all assumptions of Theorem 2, except (i i i $)$, are satisfied. Let $x(t)$ be the solution of $(1.4)-(1.5)$ and $\lim _{t \rightarrow \infty} x(t)=l\left(x_{0}, x_{-1}\right)$. If

$$
\int_{t}^{\lfloor t+1\rfloor}|a(s)| d s \leq \rho<1,
$$

then

$$
\begin{aligned}
l\left(x_{0}, x_{-1}\right) & =\left(1+\int_{0}^{1} y(s) a(s) d s\right) x_{0}+\left(\int_{0}^{1} y(s) a(s) d s\right) x_{-1} \\
& +\int_{0}^{\infty} y(s) f(s) d s+\sum_{i=1}^{\infty} d_{i}+\sum_{i=1}^{\infty}\left(z_{i}-z_{i-1}\right) \int_{i}^{i+1} y(s) a(s) d s
\end{aligned}
$$


where $y$ is a solution of the integral equation

$$
y(t)=1+\int_{t}^{\lfloor t+1\rfloor} y(s) a(s) d s, t \geq 0,
$$

and $z_{i}$ is a solution of the difference equation (2.6) for $n=i$.

\section{PROOFS}

In this section the proofs of the main results are given.

\subsection{Proof of Theorem 2.}

For the proof of Theorem 2 we consider the following well known lemma [39]:

Lemma 1. Let a nonnegative piecewise continuous function $u(t)$ satisfy for $t \geq t_{0}$ the inequality

$$
u(t) \leq \alpha+\int_{t_{0}}^{t} v(s) u(s) d s+\sum_{t_{0} \leq \tau_{i}<t} \beta_{i} u\left(\tau_{i}\right)
$$

where $\alpha \geq 0, \beta_{i} \geq 0, v(s)>0, \tau_{i}$ are the first kind discontinuity points of the function $u(t)$. Then the following estimate holds for the function $u(t)$,

$$
u(t) \leq \alpha \prod_{t_{0} \leq \tau_{i}<t}\left(1+\beta_{i}\right) \exp \left(\int_{t_{0}}^{t} v(s) d s\right) .
$$

Now we can give the proof of Theorem 2:

Proof of Theorem 2. Let $x(t)$ be the solution of (1.1)-(1.3). Then, from (2.8),

$$
\begin{aligned}
|x(t)| & \leq\left|x_{0}\right|+\int_{0}^{t}|a(s)||x(s)| d s+\int_{0}^{t}|a(s)||x(\lfloor s-1\rfloor)| d s \\
& +\int_{0}^{t}|f(s)| d s+\sum_{i=1}^{\lfloor t\rfloor}\left|c_{i}\right|\left|z_{i}\right|+\sum_{i=1}^{\lfloor t\rfloor}\left|d_{i}\right| .
\end{aligned}
$$

Considering $x(\lfloor s-1\rfloor)=z_{\lfloor s-1\rfloor}, s \in[0, \infty)$, and the boundedness of $z_{n}, n \in \mathbb{Z}^{+}$, we have

$$
|x(t)| \leq\left|x_{0}\right|+\int_{0}^{t}|a(s)||x(s)| d s+L \int_{0}^{\infty}|a(s)| d s
$$




$$
+\int_{0}^{\infty}|f(s)| d s+\sum_{i=1}^{\lfloor t\rfloor}\left|c_{i}\right|\left|z_{i}\right|+\sum_{i=1}^{\infty}\left|d_{i}\right| .
$$

By using $(i),(i i),(i v)$ and (2.7), we obtain

$$
|x(t)| \leq \alpha+\int_{0}^{t}|a(s)||x(s)| d s+\sum_{i=1}^{\lfloor t\rfloor}\left|c_{i}\right|\left|z_{i}\right|
$$

where $\alpha=\left|x_{0}\right|+L K_{1}+K_{2}+L_{2}$. Applying Lemma 1 ,

$$
\begin{aligned}
|x(t)| & \leq \alpha \prod_{i=0}^{\lfloor t\rfloor}\left(1+\left|c_{i}\right|\right) \exp \left(\int_{0}^{t}|a(s)| d s\right) \\
& \leq \alpha \prod_{i=0}^{\infty}\left(1+\left|c_{i}\right|\right) \exp \left(\int_{0}^{\infty}|a(s)| d s\right) .
\end{aligned}
$$

Hence, by $(i)$ and (iii)

$$
|x(t)| \leq \alpha L_{1} e^{K_{1}}=M, t \geq 0,
$$

where $M$ is a positive real constant.

On the other hand,

$$
\begin{aligned}
|x(t)-x(s)| & \leq \int_{s}^{t}|a(u)||x(u)| d u+\int_{s}^{t}|a(u)||x(\lfloor u-1\rfloor)| d u \\
& +\int_{s}^{t}|f(u)| d u+\sum_{i=\lfloor s\rfloor+1}^{\lfloor t\rfloor}\left|c_{i}\right|\left|z_{i}\right|+\sum_{i=\lfloor s\rfloor+1}^{\lfloor t\rfloor}\left|d_{i}\right|
\end{aligned}
$$

for $0 \leq s<t<\infty$.

From (2.7) and (4.1), we obtain

$|x(t)-x(s)| \leq(M+L) \int_{s}^{\infty}|a(u)| d u+\int_{s}^{\infty}|f(u)| d u+L \sum_{i=\lfloor s\rfloor+1}^{\infty}\left|c_{i}\right|+\sum_{i=\lfloor s\rfloor+1}^{\infty}\left|d_{i}\right|$.

In this while, we note that

$$
\sum_{i=1}^{\infty}\left|c_{i}\right|<\infty
$$

since ( $i$ i $i)$ is valid. So, by using (4.3) and the conditions $(i),(i i)$ and (iv), it is easy to verify that

$$
\lim _{s \rightarrow \infty}|x(t)-x(s)|=0
$$


Thus, by the Cauchy convergence criterion, $\lim _{t \rightarrow \infty} x(t) \in \mathbb{R}$.

\subsection{Proof of Theorem 3.}

For the proof of Theorem 3, it is necessary to prove the following theorem and lemmas.

Theorem 4. Suppose a $(t)$ is continuous and (3.1) is satisfied. Then, there is a unique bounded function $y \in P R C([0, \infty), \mathbb{R})$ such that (3.3) holds.

Proof. Denote the set of piecewise right continuous functions by $P R C([0, \infty), \mathbb{R})$, that is, $\varphi \in P R C$ means that $\varphi:[0, \infty) \rightarrow \mathbb{R}$ is continuous for $t \in[0, \infty), t \neq n, n=$ $1,2, \ldots$, and is continuous from the right for $t=n, n=0,1,2, \ldots$.

Now, let us take the space

$$
B=\left\{y \in \operatorname{PRC}([0, \infty), \mathbb{R}):|y|_{B} \leq \lambda, \lambda \geq \frac{1}{1-\rho}\right\} .
$$

$B$ is a Banach space with the norm

$$
|y|_{B}=\sup _{t \geq 0}|y(t)|, y \in B
$$

For $y \in B$ and $t \geq 0$, define

$$
T y(t)=1+\int_{t}^{\lfloor t+1\rfloor} y(s) a(s) d s .
$$

It can be easily shown that for every integer point $n, n=0,1,2, \ldots$,

$$
T y\left(n^{+}\right)=\lim _{t \rightarrow n^{+}} T y(t)=T y(n)
$$

and

$$
\operatorname{Ty}\left(n^{-}\right)=\lim _{t \rightarrow n^{-}} T y(t)=1 .
$$

As well as, for $t_{*} \in(n, n+1), n=0,1,2, \ldots$,

$$
T y\left(t_{*}^{+}\right)=T y\left(t_{*}^{-}\right)=T y\left(t_{*}\right) .
$$

So, $T y \in P R C([0, \infty), \mathbb{R})$.

Also, from (3.1), it follows that

$$
|T y|_{B} \leq 1+\rho|y|_{B} \leq \lambda .
$$

Hence $T$ maps $B$ into itself. On the other hand, for $y, z \in B$

$$
|T y-T z|_{B} \leq \rho|y-z|_{B} .
$$

Since $\rho<1, T: B \rightarrow B$ is a contraction. Therefore, the unique solution $y \in B$ of $T y=y$ is the unique piecewise right continuous and bounded solution of (3.3). 
Lemma 2. Let a $(t)$ is continuous and (3.1) is true. Then, the solution $y$ of the integral equation (3.3) satisfies the following integral equation

$$
\left\{\begin{array}{c}
y^{\prime}(t)=-y(t) a(t), t \neq n \\
\Delta y(n)=\int_{n}^{n+1} y(s) a(s) d s, n \in \mathbb{Z}^{+},
\end{array}\right.
$$

where $\Delta y(n)=y\left(n^{+}\right)-y\left(n^{-}\right), y\left(n^{+}\right)=\lim _{t \rightarrow n^{+}} y(t)$ and $y\left(n^{-}\right)=\lim _{t \rightarrow n^{-}} y(t)$.

Proof. Taking the derivative of (3.3) for $t \in(n, n+1), n \in \mathbb{Z}^{+}$, we obtain

$$
y^{\prime}(t)=-y(t) a(t) \text {. }
$$

On the other hand,

$$
\begin{aligned}
\Delta y(n) & =y\left(n^{+}\right)-y\left(n^{-}\right) \\
& =1+\int_{n}^{n+1} y(s) a(s) d s-1 \\
& =\int_{n}^{n+1} y(s) a(s) d s .
\end{aligned}
$$

So, the proof of Lemma 2 is complete.

Now, let us denote the function

$$
C(t)=y(t) x(t)-\int_{t}^{\lfloor t+1\rfloor} y(s) a(s) x(\lfloor s-1\rfloor) d s, t \geq 0,
$$

where $y$ is the solution of the integral equation (3.3) and $x$ is the solution of (1.4) (1.5).

Lemma 3. Suppose that $a(t)$ and $f(t)$ are continuous functions on $[0, \infty)$ and (3.1) is hold. Then,

$$
C(t)=C(0)+\int_{0}^{t} y(s) f(s) d s+\sum_{i=1}^{\lfloor t\rfloor} d_{i}+\sum_{i=1}^{\lfloor t\rfloor}\left(z_{i}-z_{i-1}\right) \int_{i}^{i+1} y(s) a(s) d s
$$

where $y$ is a solution of (3.3) and $z_{i}$ is a solution of the difference equation (2.6) for $n=i$.

Proof. To obtain (4.6), it is enough to show that $C(t)$, defined by (4.6), satisfies

$$
\left\{\begin{array}{c}
C^{\prime}(t)=y(t) f(t), t \neq n, t \geq 0 \\
\Delta C(n)=d_{n}+\left(z_{n}-z_{n-1}\right) \int_{n}^{n+1} y(s) a(s) d s, n \in \mathbb{Z}^{+} .
\end{array}\right.
$$


For $t \in(n, n+1),(4.5)$ can be written as

$$
C(t)=y(t) x(t)-x(n-1) \int_{t}^{n+1} y(s) a(s) d s
$$

By taking derivative of both sides of (4.8), we have

$$
C^{\prime}(t)=y^{\prime}(t) x(t)+y(t) x^{\prime}(t)+x(n-1) y(t) a(t) .
$$

Considering (1.4) and (4.4), we get

$$
\begin{aligned}
C^{\prime}(t) & =-y(t) a(t) x(t)+y(t)\{a(t) x(t)-a(t) x(n-1)+f(t)\}+x(n-1) y(t) a(t) \\
& =y(t) f(t) .
\end{aligned}
$$

Also, from (4.8),

$$
\begin{aligned}
\Delta C(n) & =C\left(n^{+}\right)-C\left(n^{-}\right) \\
& =y(n) x(n)-x(n-1) \int_{n}^{n+1} y(s) a(s) d s-y\left(n^{-}\right) x\left(n^{-}\right) .
\end{aligned}
$$

Substituting

$$
y\left(n^{-}\right)=y(n)-\int_{n}^{n+1} y(s) a(s) d s
$$

and

$$
x\left(n^{-}\right)=x(n)-d_{n}
$$

into (4.9), we obtain

$$
\Delta C(n)=d_{n}+\left(z_{n}-z_{n-1}\right) \int_{n}^{n+1} y(s) a(s) d s
$$

where $z_{n}=x(n), n \in \mathbb{Z}^{+}$. So, we obtain (4.7). Integrating both sides of (4.7), we get (4.6).

Now, we can prove Theorem 3:

Proof of Theorem 3. Let $x(t)$ be the solution of (1.4) - (1.5). For the proof, it is sufficient to show that

$$
\lim _{t \rightarrow \infty} x(t)=C(0)+\int_{0}^{\infty} y(s) f(s) d s+\sum_{i=1}^{\infty} d_{i}+\sum_{i=1}^{\infty}\left(z_{i}-z_{i-1}\right) \int_{i}^{i+1} y(s) a(s) d s
$$


where $C$ is defined by (4.5) and $z_{i}$ is a solution of the difference equation (2.6) for $n=i$. From (4.6), we have for $t \geq 0$,

$$
\begin{aligned}
& x(t)-C(0)-\int_{0}^{\infty} y(s) f(s) d s-\sum_{i=1}^{\infty} d_{i}-\sum_{i=1}^{\infty}\left(z_{i}-z_{i-1}\right) \int_{i}^{i+1} y(s) a(s) d s \\
& =x(t)-\left(C(0)+\int_{0}^{t} y(s) f(s) d s+\sum_{i=1}^{\lfloor t\rfloor} d_{i}+\sum_{i=1}^{\lfloor t\rfloor}\left(z_{i}-z_{i-1}\right) \int_{i}^{i+1} y(s) a(s) d s\right) \\
& -\int_{t}^{\infty} y(s) f(s) d s-\sum_{i=\lfloor t\rfloor+1}^{\infty} d_{i}-\sum_{i=\lfloor t\rfloor+1}^{\infty}\left(z_{i}-z_{i-1}\right) \int_{i}^{i+1} y(s) a(s) d s \\
& =x(t)-C(t)-\int_{t}^{\infty} y(s) f(s) d s-\sum_{i=\lfloor t\rfloor+1}^{\infty} d_{i}-\sum_{i=\lfloor t\rfloor+1}^{\infty}\left(z_{i}-z_{i-1}\right) \int_{i}^{i+1} y(s) a(s) d s .
\end{aligned}
$$

Using (4.5), it follows for $t \geq 0$,

$$
\begin{aligned}
& x(t)-C(0)-\int_{0}^{\infty} y(s) f(s) d s-\sum_{i=1}^{\infty} d_{i}-\sum_{i=1}^{\infty}\left(z_{i}-z_{i-1}\right) \int_{i}^{i+1} y(s) a(s) d s \\
& =x(t)-y(t) x(t)+\int_{t}^{\lfloor t+1\rfloor} y(s) a(s) x(\lfloor s-1\rfloor) d s-\int_{t}^{\infty} y(s) f(s) d s \\
& -\sum_{i=\lfloor t\rfloor+1}^{\infty} d_{i}-\sum_{i=\lfloor t\rfloor+1}^{\infty}\left(z_{i}-z_{i-1}\right) \int_{i}^{i+1} y(s) a(s) d s .
\end{aligned}
$$

On the other hand, multiplying (3.3) by $x(t)$ we obtain

$$
x(t)=y(t) x(t)-\int_{t}^{\lfloor t+1\rfloor} y(s) a(s) x(t) d s
$$

for $t \geq 0$. Substituting the last expression into (4.11), we find

$$
\begin{aligned}
& x(t)-C(0)-\int_{0}^{\infty} y(s) f(s) d s-\sum_{i=1}^{\infty} d_{i}-\sum_{i=1}^{\infty}\left(z_{i}-z_{i-1}\right) \int_{i}^{i+1} y(s) a(s) d s \\
& =\int_{t}^{\lfloor t+1\rfloor} y(s) a(s)(x(\lfloor s-1\rfloor)-x(t)) d s-\int_{t}^{\infty} y(s) f(s) d s
\end{aligned}
$$


$-\sum_{i=\lfloor t\rfloor+1}^{\infty} d_{i}-\sum_{i=\lfloor t\rfloor+1}^{\infty}\left(z_{i}-z_{i-1}\right) \int_{i}^{i+1} y(s) a(s) d s$.

From (4.12), together with (2.7), (4.1) and the boundedness of $y(t)$ on $[0, \infty)$, we get

$$
\begin{aligned}
& \left|x(t)-C(0)-\int_{0}^{\infty} y(s) f(s) d s-\sum_{i=1}^{\infty} d_{i}-\sum_{i=1}^{\infty}\left(z_{i}-z_{i-1}\right) \int_{i}^{i+1} y(s) a(s) d s\right| \\
& \leq|y|_{B}(L+M) \int_{t}^{\lfloor t+1\rfloor}|a(s)| d s+|y|_{B} \int_{t}^{\infty} f(s) d s \\
& +\sum_{i=\lfloor t\rfloor+1}^{\infty}\left|d_{i}\right|+2 L|y|_{B} \sum_{i=\lfloor t\rfloor+1}^{\infty} \int_{i}^{i+1}|a(s)| d s
\end{aligned}
$$

where $|y|_{B}=\sup _{t \geq 0}|y(t)|$. Thus, it follows that (4.10) is correct. Taking into account (4.5), it is easily verified that the limit relation (4.10) is reduced to (3.2). So, the proof is completed.

\section{ACKNOWLEDGEMENT}

We are very grateful to referee for their affirmative contributions and valuable comments.

\section{REFERENCES}

[1] A. R. Aftabizadeh and J. Wiener, "Oscillatory properties of first order linear functional differential equations," Appl. Anal., vol. 20, pp. 165-187, 1985.

[2] A. R. Aftabizadeh and J. Wiener, "Oscillatory and periodic solutions for systems of two first order linear differential equations with piecewise linear differential equations with piecewise constant argument," Appl. Anal., vol. 26, no. 4, pp. 327-333, 1988.

[3] A. R. Aftabizadeh, J. Wiener, and J.-M. Xu, "Oscillatory and periodic solutions of delay differential equations with piecewise constant argument," Proc. Am. Math. Soc., vol. 99, pp. 673-679, 1987.

[4] M. U. Akhmet, "Integral manifolds of differential equations with piecewise constant argument of generalized type," Nonlinear Anal., Theory Methods Appl., Ser. A, Theory Methods, vol. 66, no. 2, pp. 367-383, 2007.

[5] M. U. Akhmet, "On the reduction principle for differential equations with piecewise constant argument of generalized type," J. Math. Anal. Appl., vol. 336, no. 1, pp. 646-663, 2007.

[6] O. Arino and M. Pituk, "More on linear differential systems with small delays," J. Differ. Equations, vol. 170, no. 2, pp. 381-407, 2001. 
[7] D. D. Bainov and P. S. Simeonov, Impulsive differential equations. Asymptotic properties of the solutions, ser. Advances in Mathematics for Applied Sciences. Singapore: World Scientific, 1995, vol. 28.

[8] J. Baštinec, J. Diblík, and Z. Šmarda, "Convergence tests for one scalar differential equation with vanishing delay," Arch. Math., Brno, vol. 36, pp. 405-414, 2000.

[9] H. Bereketoglu, G. Seyhan, and A. Ogun, "Advanced impulsive differential equations with piecewise constant arguments," Math. Model. Anal., vol. 15, no. 2, pp. 175-187, 2010.

[10] H. Bereketoglu and F. Karakoç, "Asymptotic constancy for impulsive delay differential equations," Dyn. Syst. Appl., vol. 17, no. 1, pp. 71-83, 2008.

[11] H. Bereketoglu and M. Pituk, "Asymptotic constancy for nonhomogeneous linear differential equations with unbounded delays," Discrete Contin. Dyn. Syst., vol. 2003, pp. 100-107, 2003.

[12] L. Berezansky, J. Diblík, M. Růžičková, and Z. Šutá, "Asymptotic convergence of the solutions of a discrete equation with two delays in the critical case," Abstr. Appl. Anal., vol. 2011, p. 15, 2011.

[13] S. Busenberg and K. L. Cooke, "Models of vertically transmitted diseases with sequentialcontinuous dynamics," in Nonlinear phenomena in mathematical sciences, Proc. int. Conf., Arlington/Tex. 1980, 1982, pp. 179-187.

[14] K. L. Cooke and J. Wiener, "Retarded differential equations with piecewise constant delays," J. Math. Anal. Appl., vol. 99, pp. 265-297, 1984.

[15] J. Diblík, "A criterion of asymptotic convergence for a class of nonlinear differential equations with delay," Nonlinear Anal., Theory Methods Appl., Ser. A, Theory Methods, vol. 47, no. 6, pp. 4095-4106, 2001.

[16] J. Diblík, M. Růžičková, Z. Šmarda, and Z. Šutá, "Asymptotic convergence of the solutions of a dynamic equation on discrete time scales," Abstr. Appl. Anal., vol. 2012, p. 20, 2012.

[17] J. Diblík, M. Růžičková, and Z. Šutá, "Asymptotic convergence of the solutions of a discrete equation with several delays," Appl. Math. Comput., vol. 218, no. 9, pp. 5391-5401, 2012.

[18] J. Diblík, M. Rǔžičková, and Z. Šutá, "Asymptotic convergence of the solutions of a discrete system with delays," Appl. Math. Comput., vol. 219, no. 8, pp. 4036-4044, 2012.

[19] J. Diblík, "Behaviour of solutions to linear differential equations with delay," Arch. Math., Brno, vol. 34, no. 1, pp. 31-47, 1998.

[20] J. Diblík, "A criterion for convergence of solutions to homogeneous delay linear differential equations," Ann. Pol. Math., vol. 72, no. 2, pp. 115-130, 1999.

[21] J. Diblík, "Asymptotic convergence criteria of solutions of delayed functional differential equations," J. Math. Anal. Appl., vol. 274, no. 1, pp. 349-373, 2002.

[22] J. Diblík and M. Růžičková, "Convergence of the solutions of the equation $\dot{y}(t)=\beta(t)[y(t-\delta)-$ $y(t-\tau)]$ in the critical case," J. Math. Anal. Appl., vol. 331, no. 2, pp. 1361-1370, 2007.

[23] J. Diblík, M. Růžičková, and Z. Šutá, "Asymptotical convergence of the solutions of a linear differential equation with delays," Adv. Difference Equ., vol. 2010, p. 12, 2010.

[24] R. K. George, A. K. Nandakumaran, and A. Arapostathis, "A note on controllability of impulsive systems," J. Math. Anal. Appl., vol. 241, no. 2, pp. 276-283, 2000.

[25] Z.-H. Guan, G. Chen, and T. Ueta, "On impulsive control of a periodically forced chaotic pendulum system,” IEEE Trans. Autom. Control, vol. 45, no. 9, pp. 1724-1727, 2000.

[26] I. Györi and G. Ladas, "Linearized oscillations for equations with piecewise constant arguments," Differ. Integral Equ., vol. 2, no. 2, pp. 123-131, 1989.

[27] I. Györi, "On approximation of the solutions of delay differential equations by using piecewise constant arguments," Int. J. Math. Math. Sci., vol. 14, no. 1, pp. 111-126, 1991.

[28] Y. K. Huang, "Oscillations and asymptotic stability of solutions of first order neutral differential equations with piecewise constant argument," J. Math. Anal. Appl., vol. 149, no. 1, pp. 70-85, 1990. 
[29] F. Karakoc, H. Bereketoglu, and G. Seyhan, "Oscillatory and periodic solutions of impulsive differential equations with piecewise constant argument," Acta Appl. Math., vol. 110, no. 1, pp. 499-510, 2010.

[30] F. Karakoç and H. Bereketoglu, "Some results for linear impulsive delay differential equations," Dyn. Contin. Discrete Impuls. Syst., Ser. A, Math. Anal., vol. 16, no. 3, pp. 313-326, 2009.

[31] A. Lakmeche and O. Arino, "Bifurcation of non trivial periodic solutions of impulsive differential equations arising chemotherapeutic treatment," Dyn. Contin. Discrete Impulsive Syst., vol. 7, no. 2, pp. 265-287, 2000.

[32] S. Lenci and G. Rega, "Periodic solutions and bifurcations in an impact-inverted pendulum under impulsive excitation," Chaos Solitons Fractals, vol. 11, no. 15, pp. 2453-2472, 2000.

[33] J. Li and J. Shen, "Periodic boundary value problems of impulsive differential equations with piecewise constant argument," J. Nat. Sci. Hunan Norm. Univ., vol. 25, no. 3, pp. 5-9, 2002.

[34] X. Li, "Impulsive stabilization of certain delay differential equations with piecewise constant argument," Int. J. Difference Equ., vol. 3, no. 2, pp. 267-276, 2008.

[35] H. Liang and G.-Q. Wang, "Existence and uniqueness of periodic solutions for a delay differential equation with piecewise constant arguments," Port. Math. (N.S.), vol. 66, no. 1, pp. 1-12, 2009.

[36] Y. Muroya, "New contractivity condition in a population model with piecewise constant arguments," J. Math. Anal. Appl., vol. 346, no. 1, pp. 65-81, 2008.

[37] S. Nenov, "Impulsive controllability and optimization problems in population dynamics," Nonlinear Anal., Theory Methods Appl., Ser. A, Theory Methods, vol. 36, no. 7, pp. 881-890, 1999.

[38] M. Pinto, "Asymptotic equivalence of nonlinear and quasi linear differential equations with piecewise constant arguments," Math. Comput. Modelling, vol. 49, no. 9-10, pp. 1750-1758, 2009.

[39] A. M. Samoilenko and N. A. Perestyuk, Impulsive differential equations, ser. World Scientific Series on Nonlinear Science. Series A. Singapore: World Scientific, 1995, vol. 14.

[40] S. M. Shah and J. Wiener, "Advanced differential equations with piecewise constant argument deviations," Int. J. Math. Math. Sci., vol. 6, pp. 671-703, 1983.

[41] J. Čermák, "Asymptotic behaviour of solutions to some differential equations with an unbounded delay," Electron. J. Qual. Theory Differ. Equ., vol. 2000, p. 8, 2000.

[42] J. Wiener, "Differential equations with piecewise constant delays," in Trends in theory and practice of nonlinear differential equations, Proc. int. Conf., Arlington/Tex. 1982, ser. Lect. Notes Pure Appl. Math., 1984, vol. 90, pp. 547-552.

[43] J. Wiener, Generalized solutions of functional differential equations. Singapore: World Scientific, 1993.

[44] J. Wiener and V. Lakshmikantham, "Differential equations with piecewise constant argument and impulsive equations," Nonlinear Stud., vol. 7, no. 1, pp. 60-69, 2000.

\section{Authors' addresses}

\section{Huseyin Bereketoglu}

Ankara University, Faculty of Sciences, Department of Mathematics, TR-06100 Besevler, Ankara, Turkey

E-mail address: bereket@science.ankara.edu.tr

\section{Gizem S. Oztepe}

Ankara University, Faculty of Sciences, Department of Mathematics, TR-06100 Besevler, Ankara, Turkey

E-mail address: gseyhan@ankara.edu.tr (Corresponding Author) 